\title{
Braucht eine Verfassung soziale Grundrechte? \\ Überlegungen zur koreanischen Verfassung vom 17. Juli 1948
}

\author{
Von Il Sun Hong, Seoul
}

\section{Einleitung}

Das koreanische Rechtssystem steht seit jeher unter starkem Einfluss des deutschen Rechtssystems. Häufig lassen sich für koreanische Rechtsfragen und die Fortentwicklung des koreanischen Rechts Parallelen in Deutschland finden.

Ein Beispiel dafür sind die sozialen Grundrechte in der koreanischen Verfassung. Dazu vorausgeschickt einige grundsätzliche Bemerkungen:

Schon etwa Mitte des 19. Jahrhunderts mit der beginnenden Industrialisierung hatte sich in Europa die Frage nach sozialen Grundrechten gestellt. Man gelangte zu der Erkenntnis, auch eine Verfassung solle sich den veränderten sozialen Verhältnissen nicht verschließen. Das war die Geburtsstunde sozialer Grundrechte, die sodann Eingang in etliche europäische Verfassungen fanden. Mitte des 20. Jahrhunderts schlug diese Sichtweise dann um. Man fragte: Muss wirklich eine Verfassung soziale Grundrechte enthalten? Können sie soziale Probleme lösen? Notwendigerweise deuten diese Fragen auf die Natur dieser Art von Grundrechten.

Der Begriff „soziale Grundrechte“ konnte noch nicht allgemein verbindlich definiert werden. Er ist noch immer streitig. Einig ist man sich nur über den strukturellen Unterschied zwischen Sozialgrundrechten und Freiheitsgrundrechten: Während Freiheitsgrundrechte etwas bereits Vorhandenes vor Eingriffen schützen, zielen soziale Grundrechte auf die Schaffung oder Zuteilung von etwas, über das der Anspruchsteller noch nicht verfügt. ${ }^{1}$ Es geht im Grunde also um die ,verfassungsrechtliche Verankerung eines Leistungsanspruchs“. 2 
Einig ist man sich ferner, dass rechtliche Freiheit ohne faktische Realisierbarkeit zwecklos ist. $^{3}$ Dies namentlich im Bereich der sozialen Grundrechte: Ohne beispielsweise die Möglichkeit, Arbeit zu finden, läuft das Grundrecht auf Berufsausübungsfreiheit leer. Insofern lässt sich sozialen Grundrechte auch eine Funktion zuerkennen: Sie leisten die „effektive Ermöglichung von Freiheitsgrundrechten“.

Die folgenden Überlegungen zum koreanischen "Import" sozialer Grundrechte aus Deutschland nehmen ihren Ausgang kurz bei der Weimarer Reichsverfassung vom 11. August 1919 (II), widmen sich dann der geltenden koreanischen Verfassung (III) und blicken zuletzt auf das Grundgesetz, eine Verfassung ohne ausdrückliche soziale Grundrechte (IV).

\section{Soziale Grundrechte in der Weimarer Reichsverfassung}

Typische soziale Grundrechte sind neben dem klassischen Recht auf Arbeit etwa das Recht auf Fürsorge bei Krankheit oder Armut sowie die Rechte auf Bildung und auf Wohnung. Diese typischen sozialen Grundrechte erwähnte in Deutschland zum ersten Mal die Weimarer Reichsverfassung. Die sozialen Grundrechte in der Weimarer Reichsverfassung waren jedoch in mehrfacher Hinsicht problematisch. ${ }^{4}$

Erstens waren die Formulierungen vielfach verschwommen. Sie ließen mehrere Interpretationsmöglichkeiten zu. Beispielhafte Formulierungen: Die Jugend ,ist zu schützen“ (Art. 122 WRV); das Volksbildungswesen ,soll gefördert werden“ (Art. 148 WRV). Nur beim Recht auf Arbeit formulierte die Verfassung konkreter: "Jedem Deutschen soll die Möglichkeit gegeben werden, durch wirtschaftliche Arbeit seinen Unterhalt zu erwerben" (Art. 163 Abs. 2, Satz 1 WRV).

Zweitens kleidete die Weimarer Reichsverfassung soziale Grundrechte überwiegend in Gesetzgebungsaufträge. Die sozialen Grundrechte bedurften einer Konkretisierung durch entsprechende Gesetze. Deren Zahl ist indes gering geblieben. Es konnten nur Leistungsgesetze sein, und die politischen und wirtschaftlichen Verhältnisse der damaligen Zeit waren nicht dazu angetan, hier aus dem Vollen zu schöpfen.

Die unmittelbare rechtliche Wirkung sozialer Grundrechte in der Weimarer Reichsverfassung war deshalb schwach. Herrschende Meinung und Rechtsprechung in der Weimarer 
Zeit sahen bereits die Grundrechte allgemein nur als „Programmsätze“. ${ }^{5}$ Erst recht konnten deshalb soziale Grundrechte den Gesetzgeber nicht zur Gesetzgebung verpflichten. Lediglich als „Programme“ ohne Rechtssubstanz verstanden, vermochten sie letztlich nicht hinauszugelangen über den Rang rechtlich unverbindlicher Absichtserklärungen.

Trotzdem verdient die Bedeutung der Weimarer Reichsverfassung für die weitere verfassungsrechtliche Entwicklung sozialer Grundrechte keine Geringschätzung. Insbesondere auf die Regelungen über soziale Grundrechte in den bundesrepublikanischen Landesverfassungen bis in die neuen Landesverfassungen nach 1990 hat die Weimarer Reichsverfassung großen Einfluss entfaltet. ${ }^{6}$

\section{Soziale Grundrechte in der koreanischen Verfassung}

Als sich Korea am 17. Juli 1948 seine erste Verfassung gab, war das Grundgesetz noch in Arbeit. Das musste den Blick der traditionell am deutschen Rechtssystem interessierten koreanischen Verfassungsgeber zwangsläufig auf die Weimarer Reichsverfassung lenken. Ihr wuchs, obwohl kein geltendes Recht mehr, gleichsam die Rolle eines Paten für die erste koreanische Verfassung zu, ungeachtet der wenig ermutigenden Weimarer Verfassungswirklichkeit. Die koreanische Verfassung nahm in ihren Grundrechtskatalog neben den sozialen Grundrechten der Weimarer Verfassung noch weitere auf. Kaum eines der damals wie heute bekannten sozialen Grundrechte, das sich in der koreanischen Verfassung nicht findet. Trotz neunmaliger Verfassungsänderung sind diese sozialen Grundrechte bis jetzt kaum geändert worden.

Die geltende koreanische Verfassung gewährleistet insgesamt an die elf soziale Grundrechte, darunter namentlich die Rechte auf Bildung, ${ }^{8}$ auf Arbeit, ${ }^{9}$ auf Schutz von Ehe und

Frotscher/Pieroth, Verfassungsgeschichte, 274; Anschütz, Die Verfassung des Deutschen Reichs vom 11. August 1919, 13. Aufl. 1930, S. 453 ff.

Kutscha, ZRP 1993, 339 (339 ff.); Maurer, Staatsrecht I, 240 ff.

7 Zu den sozialen Grundrechten in der koreanischen Verfassung siehe vor allem Seong-Bang Hong, Soziale Rechte auf der Verfassungsebene und auf der gesetzlichen Ebene. Unter besonderer Berücksichtigung der sozialen Rechte im Grundgesetz der Bundesrepublik Deutschland, in der Verfassung der Republik Korea und im deutschen Sozialgesetzbuch, Diss. Uni. Köln, 1986; Vgl. auch Soen-Taek Kim, Die Interpretation der Grundrechte nach dem Grundgesetz für die Bundesrepublik Deutschland und nach der Verfassung der Republik Korea, Diss. Uni. Köln, 1992.

Art. 31 Abs. 1 KV: „Jeder Staatsbürger hat das Recht, entsprechend seinen Fähigkeiten gleichberechtigt Bildung zu genießen.“

9

Art. 32 Abs. $1 \mathrm{KV}$ : „Jeder Staatsbürger hat das Recht auf Arbeit. Der Staat hat auf soziale und wirtschaftliche Weise für eine Steigerung des Beschäftigungsstandes der Arbeitnehmer und für die 
Familie, ${ }^{10}$ auf ein menschenwürdiges Leben, ${ }^{11}$ auf eine saubere Umwelt und auf Gesundheit. $^{12}$

Geht man diese Bestimmungen durch, erkennt man ohne Schwierigkeit, welche von ihnen der Weimarer Reichsverfassung entlehnt sind. Es wundert deshalb nicht, dass der wissenschaftliche Grundrechtsdiskurs in Korea auch heute noch weitgehend den Argumentationslinien der Weimarer Zeit folgt.

Auch in Korea fragt man sich, welcher Rechtscharakter den sozialen Grundrechten zukommen soll. ${ }^{13}$ Eine Regelung wie „Jeder Staatsbürger hat das Recht auf (etwas)“", suggeriert die Gewährleistung als subjektives Recht. Gleichwohl besteht Einigkeit darüber, dass es sich nicht um einen konkreten Anspruch handeln kann. Die drei in Deutschland geläufigen Meinungsvarianten lassen sich auch in der koreanischen Diskussion ausmachen:

Die einen behaupten, soziale Grundrechte seien ausschließlich Programmsätze. Soziale Grundrechte richten sich danach als Politikziele an Gesetzgebung und Regierung. Sie sind aber gerichtlich nicht einklagbar. Dem Staatsbürger kämen also aus den Vorschriften über soziale Grundrechte keine konkreten Rechte zu.

Die zweite Meinung versteht soziale Grundrechte als sog. ,abstrakte Rechte“. Danach seien soziale Grundrechte nur in der Regel Programmsätze. Sie könnten aber ausnahmsweise auch subjektive Rechte begründen, wenn sie durch die Gesetzgebung umgesetzt würden. Das mache ihren abstrakten Charakter aus. ${ }^{15}$

Garantie eines gerechten Lohnes zu sorgen, und nach Maßgabe der Gesetze die Mindestlohnpolitik durchzuführen."

Art. 36 Abs. $1 \mathrm{KV}$ : „Ehe und Familie müssen auf die Würde des Individuums und die Gleichberechtigung der Ehepartner gegründet und erhalten werden, und der Staat gewährleistet dies.“ Art. 34 Abs. 1 KV: ,Jeder Staatsbürger hat das Recht, ein menschenwürdiges Leben zu führen.“

Art. 36 Abs. 3 KV: "Die Gesundheit aller Staatsbürger steht unter dem besonderem Schutz des Staates."

Zur diesen Problematik siehe vor allem Hee - Yol Kay, Verfassungslehre II, 2004 Pakyoungsa, 688 ff. Vgl. auch Tae - Ho Chung, Soziale Grundrechte als Prinzip, in: Festschrift für Kyoung Sik Chung zum 60. Geburtstag, 1997238 (238 ff.); ders., Soziale Grundrechte und die Rechtsprechung des koreanischen Verfassungsgerichts, Hunbubnonchong 9, 1998, 601 (601 ff.); Zunil Yi, Soziale Grundrechte, Forschung der Verfassungslehre 10/1, 2004, 449 (449 ff.). So etwa Hong-Ju Mun, Die koreanische Verfassung, 1995, 302. sozialer Grundrechte, Honbubnonchong 2 (1991), 177 (200). 
Früher war dies die herrschende Meinung. Sie ist aber problematisch. Denn diese Meinung ist im Grunde genommen nichts anderes als die erste Meinung, also eine „Programmsatztheorie“. Was ,,abstrakt“ bedeutet, ist nur schwer einsichtig. Es stellt sich zudem die Frage, ob so verstanden ein abstraktes Recht überhaupt ein "Recht" ist.

Nach der dritten Meinung sind soziale Grundrechte „Staatszielbestimmungen“. Diese Meinung versteht die sozialen Grundrechte in der koreanischen Verfassung als Ausdruck des „Sozialstaatsprinzips“, wie im Sinne des deutschen Grundgesetzes. Soziale Grundrechte verpflichten danach den Staat zu ihrer Beachtung und Erfüllung. Sie richten sich aber in erster Linie an den Gesetzgeber. In dessen Regelungen konkretisiert, seien sie gerichtlich durchsetzbar. Aber diese Möglichkeit beschränkt sich auf die Fälle evidenter, offensichtlicher Verstöße. Denn der Gesetzgeber hat einen Gestaltungsspielraum. Heute ist in Korea dies die herrschende Meinung. ${ }^{16}$

Auch das koreanische Verfassungsgericht, das seit 1988 besteht, versteht soziale Grundrechte als „Staatszielbestimmungen.“ So hat das Gericht in seiner Entscheidung zum Recht auf ein menschenwürdiges Leben ausgesprochen, dass dieses Recht den Staat zu seiner Beachtung und Erfüllung verpflichte. Die Untätigkeit oder grobe Vernachlässigung dieser Pflicht des Staates könne subjektiv-rechtlich geltend gemacht werden. ${ }^{17}$

Auch diese sog. „Staatszielbestimmungstheorie“ stieß aber auf Kritik. Damit haben weder die Rechtslehre noch die Rechtsprechung in Korea auf die Einordnungsprobleme von sozialen Grundrechten eine eindeutige Antwort gegeben. ${ }^{18}$

\section{Soziale Grundrechte im Grundgesetz}

Angesichts dieser Einordnungsprobleme und vor dem Hintergrund einer möglichen Einheitsverfassung für Nord- und Süd- Korea stellt sich in Korea derzeit die Frage, ob eine solche Einheitsverfassung soziale Grundrechte normieren sollte. Auch in Deutschland

So etwa Yong-Su Chang, Grundrechte 2000, 525; Soo-Eung Han, Die Schranke der Verwirklichung sozialer Grundrechte im Wege der Verfassungsgerichtsbarkeit, Gerechtigkeit und Menschenrechte, 1997, 70 (75).

Entscheidungen des koreanischen Verfassungsgerichts 9-1, 543 (544). Vgl. auch Entscheidungen des koreanischen Verfassungsgerichts 11-2, 732 (732); 15-2, 179 (179 ff.).

18

Neuerdings, in Anlehnung an die Auffassung von Alexy, behaupten einige Autoren, dass die sozialen Grundrechte in der koreanischen Verfassung als "Prinzipien" verstanden werden können. So etwa Hee-Yol Kay, Die Verfassungslehre II, 698 f; Tae - Ho Chung, Soziale Grundrechte als Prinzip, in: Festschrift für Kyoung - Sik Chung zum 60. Geburtstag, 1997238 (245); Zunil Yi, Soziale Grundrechte, Forschung der Verfassungslehre 10/1, 2004, 449 (449 ff.). 
wurde - insbesondere nach der Wiedervereinigung - darüber diskutiert. ${ }^{19}$ Man erinnert sich an die Auseinandersetzungen in den Vereinten Nationen über eine einheitliche UNMenschenrechtskodifikation. Nur getrennt konnten sich schließlich der Westen mit dem Pakt über bürgerliche und politische Rechte und der Osten mit dem Pakt über wirtschaftliche, soziale und kulturelle Rechte durchsetzen ${ }^{20}$.

Der Grundgesetzgeber hat bewusst auf die Festlegung sozialer Grundrechte verzichtet, wenn man einmal absieht vom Schutzanspruch von Ehe und Familie (Art. 6 Abs. 1 GG). An Stelle weiterer sozialer Grundrechte formuliert das Grundgesetz objektiv-rechtliche Aussagen wie „sozialer Bundesstaat“ (Art. 20 I GG) und „,sozialer Rechtsstaat“ (Art. 28 I GG). Trotzdem hat das Grundgesetz durch die Grundrechtsinterpretation eine Auslegung erfahren, die den sozialen Grundrechten in manchem nahe kommt.

Dabei hat das Bundesverfassungsgericht eine entscheidende Rolle gespielt. Das Bundesverfassungsgericht bleibt in ständiger Rechtsprechung dabei, dass Grundrechte nicht nur subjektive Abwehrrechte sind, sondern zugleich eine objektive Wertordnung verkörpern. ${ }^{21}$ Dies verpflichtet den Staat, zur Grundrechtsverwirklichung beizutragen. Im Zusammenhang damit hat das Bundesverfassungsgericht dem Sozialstaatsprinzip, wenn auch nur in begrenztem Umfang, konkrete staatliche Handlungspflichten entnommen. Zum Beispiel wurde aus Art. 1 Abs. 1 GG im Zusammenhang mit dem Sozialstaatsprinzip die „Pflicht des Staates“ hergeleitet, das individuelle Existenzminimum zu sichern. ${ }^{22}$ Auch die Rechtsprechung zu den sog. Teilhaberechten wäre hier zu nennen. ${ }^{23}$ So hat sich gezeigt, dass die Verfassung auch ohne soziale Grundrechte soziale Anliegen anerkennt. ${ }^{24}$

\section{Schluss}

Die Erfahrungen mit sozialen Grundrechten in der Weimarer Zeit, in Korea und in der Bundesrepublik Deutschland zeigen: Die Besonderheit sozialer Grundrechte liegt darin, dass sie der Ausgestaltung durch den Gesetzgeber bedürfen. Soziale Grundrechte beziehen sich auf eine stets veränderliche Realität. Konkrete Formulierungen, die einer Situation

Vgl. dazu etwa Bergmann/Hermann, JA 1992, 337 (337 ff.); zuvor Isensee, Der Staat 19 (1980), 367 (367 ff.) BVerfGE 33, 303 (333); 43, 291 (313 ff.). Vgl. auch Hesse, Grundzüge des Verfassungsrechts, Rn. 289.

24 So auch Isensee, Der Staat 19 (1980), 367 (383). 
angepasst sind, werden rasch durch die Veränderungen der Zeit überholt. Hinzu kommt: Die effektive Geltung sozialer Grundrechte hängt auch von der Wirtschaftskonjunktur ab. In guten Zeiten sind sie durchsetzbar, in Zeiten der wirtschaftlichen Krise kaum.

Im Hinblick auf diese Eigenart sollte die Verwirklichung sozialer Postulate dem Parlament überlassen bleiben. Sozialpolitische Entscheidungen sind nicht die Aufgabe der Justiz, sondern des Parlaments. Wenn die Verfassung eine ausdrückliche Bestimmung über soziale Grundrechte enthält, muss sie ständig der veränderten Entwicklung angepasst werden. Sonst setzt sie sich dem Vorwurf aus, sie gebe mit ihren sozialen Garantien nur leere Versprechungen ab. Dies hat sich in der Kritik an der Weimarer Reichsverfassung ebenso wie an der koreanischen Verfassung gezeigt.

Eine Verfassung braucht nicht notwendigerweise eine ausdrückliche Normierung sozialer Grundrechte. Die Risiken überwiegen gegenüber den Vorteilen. 